\section{Zucchini has bite}

The protein Zucchini belongs to the phospholipase D (PLD) family of phosphodiesterases and has been implicated in promoting primary PIWI-interacting RNA (piRNA) biogenesis. PLD family members include both phospholipases and nucleases, but it has been unclear whether

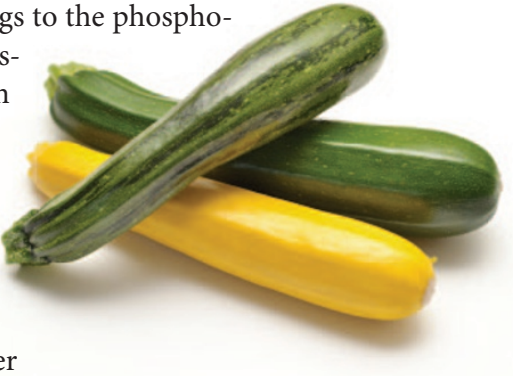
Zucchini has an impact on the piRNA biogenesis pathway indirectly (through a putative phospholipase activity) or directly (as a putative nuclease). Three recent reports address this question by analyzing mouse and Drosophila melanogaster Zucchini (mZuc and DmZuc, respectively) using structural and functional approaches. Ipsaro et al. determined the crystal structure of $\mathrm{N}$-terminally truncated mZuc, whereas Nishimasu et al. and Voigt et al. solved the structure of an equivalent truncation of DmZuc. The papers demonstrate that Zucchini forms a dimer, with the active site assembled from conserved residues of both monomers, consistent with known monomeric and dimeric PLD protein structures. The mZuc structure contains an unexpected $\mathrm{CCCH}$ type zinc-finger motif, which has been implicated in the binding of single-stranded RNA molecules, whereas DmZuc has a CHCC-type zinc-finger motif. In addition, the active site forms a narrow groove that can accommodate single-stranded, but not double-stranded, nucleic acids. Ipsaro et al. modeled a short RNA molecule into the structure of mZuc, illustrating the shape and charge complementarity provided by the RNA substrate. In support of the structural data, in vitro analyses confirmed that mZuc and DmZuc lacked any detectable phospholipase activity but instead demonstrated single-strand-specific nuclease activity. Nishimasu et al. further validated the functional significance of DmZuc's nuclease activity by demonstrating that wild-type dimeric DmZuc is critical for transposon silencing. mZuc and DmZuc cleavage generates $5^{\prime}$ phosphate and $3^{\prime}$ hydroxyl termini, which suggests that Zucchini might generate the $5^{\prime}$ ends of mature primary piRNAs, which are known to bear a $5^{\prime}$-monophosphate group. Future studies will be needed to pinpoint Zucchini's precise role in primary piRNA biogenesis. (Nature doi:10.1038/nature11502 and 10.1038/nature11509, published online 14 October 2012; RNA doi:10.1261/rna.034967.112, published online 19 October 2012)

AH

\section{Pause-itively engaged}

RNA polymerase II (Pol II) pausing downstream of gene promoters has emerged as a widespread phenomenon in eukaryotic gene expression. Pausing could prime signal-responsive genes for rapid activation; alternatively, it might serve as a checkpoint that allows recruitment of the RNA-processing machinery. Although the phenomenon-originally described 20 years ago in Drosophila melanogaster heat-shock genes-has gained acceptance as a core element of transcription in higher eukaryotes, the status of Pol II at the pause site has remained ambiguous. In a recent study, Core et al. address the transcriptional status of promoter-associated Pol II by using global run-on sequencing (GRO-seq). This method, which relies on the detection of nascent RNA, allows precise determination of the position, amount and orientation of transcriptionally engaged polymerases genome wide. By producing matched GRO-seq data sets in the presence or absence of Sarkosyl and quantitatively comparing those to Pol II ChIP-chip and ChIP-seq results, the authors confirm that a high degree of stable pausing occurs near promoters and show that most, if not all, of the promoter-associated polymerase is in a transcriptionally engaged state rather than being part of a postrecruitment preinitiation complex. In conjunction with several earlier studies, this work cements the dynamic state of the paused polymerase complex as a key tunable element of gene expression regulation. (Cell Rep. doi:10.1016/j.celrep.2012.08.034, published online 25 October 2012)

\section{A PrePPI way to make predictions}

The construction of protein-protein interactomes is an important step toward understanding cell developmental and regulatory programs. Protein-protein interactions (PPIs) have been examined by using high-throughput experimental techniques, such as yeast two-hybrid assays and affinity purification, and by computation-based approaches, including gene ontology and phylogenetic profiles. Now, Zhang et al. have developed an approach called PrePPI (predicting protein-protein interactions) that combines structural- and nonstructural-interaction data to predict PPIs on a genome-wide scale. PrePPI performs structural modeling on a pair of query proteins, making predictions based on close structural neighbors as well as homology models and remote structural relationships, greatly expanding the number of interactions that can be modeled. The resulting PPI model is scored and, by using a Bayesian network, a likelihood ratio is calculated that incorporates nonstructural evidence, such as coexpression levels and functional similarities. The authors used PrePPI to score all possible pairwise interactions in the yeast and human proteomes, predicting about 31,400 high-confidence interactions for yeast and nearly 318,000 for humans. Nineteen predictions were experimentally validated by using coimmunoprecipitation assays. One set of predictions involved potential PPIs formed by suppressor of cytokine signaling 3 (SOCS3). SOCS3's inhibitory function had already been established in the JAKSTAT pathway, but PrePPI predicted interactions with GRB2 and RAG3, components of the RAS-MAPK pathway, which were experimentally confirmed. PrePPI performs better than methods that rely on structural or nonstructural evidence alone. The authors attribute its relative success to the use of both close and remote structural neighbors, an efficient scoring strategy for their interaction models that allows them to examine a very large number of models, and the use of a Bayesian network to evaluate the predicted interaction models. Because PrePPI relies on structural representatives of sequence families, it falls short in mapping membrane protein interactions. It also presently does not take posttranslational modifications into account. However, PrePPI offers a feasible alternative to high-throughput methodologies while also providing a rough model for what a predicted PPI may look like. (Nature doi:10.1038/nature11503, published online 30 September 2012) 